\title{
Role of connectivity in congestion and decongestion in a communication network
}

\author{
N. Gupte ${ }^{1, a}$ and B.K. Singh ${ }^{2, b}$ \\ 1 Department of Physics, Indian Institute of Technology-Madras, Chennai-600 036, India \\ 2 Institute of Physics, Academia Sinica, Taipei 11529, Taiwan
}

Eur. Phys. J. B 50, 227 (2006) (DOI: 10.1140/epjb/e2006-00119-8)

Received 10 May 2006

Published online 31 May 2006 - (c) EDP Sciences, Società Italiana di Fisica, Springer-Verlag 2006

An error occurred in the author's Present Address: The address which appears under footnote a (Dept. of Infectious Diseases, Imperial College) is the Present Address of the second author (B.K. Singh) and not that of the first author (N. Gupte).

\footnotetext{
${ }^{\text {a }}$ e-mail: gupte@physics.iitm.ac.in

b Present address: Dept. of Infectious Disease Epidemiology,

St. Mary's Campus, Imperial College, London W2 1PG, UK
} 